\title{
Temporal solitons in free-space femtosecond enhancement cavities
}

\author{
N. Lilienfein ${ }^{1,2 \star}$, C. Hofer ${ }^{1,2}$, M. Högner ${ }^{1,2}$, T. Saule ${ }^{1,2}$, M. Trubetskov', V. Pervak ${ }^{2}$, E. Fill2 ${ }^{2}$, C. Riek ${ }^{3}$, \\ A. Leitenstorfer ${ }^{3}$, J. Limpert ${ }^{4}$, F. Krausz ${ }^{1,2}$ and I. Pupeza ${ }^{1,2 \star}$
}

\begin{abstract}
Temporal dissipative solitons in nonlinear optical resonators are self-compressed, self-stabilizing and indefinitely circulating wave packets. Owing to these properties, they have been harnessed for the generation of ultrashort pulses and frequency combs in active and passive laser architectures, including mode-locked lasers ${ }^{1-4}$, passive fibre resonators ${ }^{5}$ and microresonators ${ }^{6-11}$. Here, we demonstrate the formation of temporal dissipative solitons in a free-space enhancement cavity with a Kerr nonlinearity and a spectrally tailored finesse. By locking a 100-MHz-repetition-rate train of 350-fs, 1,035-nm pulses to this cavity-soliton state, we generate a 37-fs sech ${ }^{2}$-shaped pulse with a peak-power enhancement of 3,200, which exhibits low-frequency intensity-noise suppression. The power scalability unique to free-space cavities, the unprecedented combination of peak-power enhancement and temporal compression, and the cavity-soliton-specific noise filtering attest to the vast potential of this platform of optical solitons for applications including spatiotemporal filtering and compression of ultrashort pulses and cavity-enhanced nonlinear frequency conversion.
\end{abstract}

Temporal optical solitons are localized electromagnetic wave packets propagating through a dispersive, nonlinear medium while maintaining a constant structure. In nonlinear optical resonators they manifest as temporal dissipative solitons ${ }^{4}$, that is, asymptotically stable steady states associated with one or more pulses capable of circulating, in principle, ad infinitum. The duration and peak intensity of these pulses are determined by the balance between dispersion and self-phase-modulation on the one hand, and between loss and gain mechanisms on the other. Besides being highly interesting objects from a fundamental nonlinear-optics perspective, their properties, applied in particular for the passive mode-locking of active laser resonators ${ }^{1-4}$, have made them a cornerstone of ultrafast-optics and frequency-comb technology. More recently, the generation of temporal cavity solitons (CS) in externally excited, passive optical resonators has rapidly developed into an active field of research. First demonstrated in 2010 in continuous-wave (CW) laser-driven passive fibre resonators ${ }^{5}$, temporal CS formation has since been observed in various types of high-finesse microresonator $^{6-11}$, playing a crucial part in the development of a new family of frequency-comb sources ${ }^{12}$, and facilitating fundamental research on soliton dynamics ${ }^{13,14}$.

Here we report the generation of CS in a macroscopic, freespace femtosecond enhancement cavity $(\mathrm{EC})^{15-21}$ including a thin Kerr medium. In contrast to previous CS platforms, here the pulse experiences nonlinearity, dispersion correction, transverse mode guiding and energy input coupling in discrete, sequential steps. Furthermore, we demonstrate experimentally and numerically that spectrally tailoring the transmission of the input coupling mirror (ICM) to match the driving laser spectrum circumvents the limitation set by the temporal and spectral overlap between intracavity solitons and driving pulses affecting the coupling efficiency of previous CS implementations ${ }^{11}$. The resulting peak-power enhancement exceeds that demonstrated in conventional, broadband femtosecond ECs for similar pulse durations by more than one order of magnitude ${ }^{15,22}$. The intracavity pulse represents by far the most energetic CS reported to date.

The $100-\mathrm{MHz}$ bowtie ring EC consists of four mirrors with dielectric coatings, and an uncoated sapphire plate (Brewster plate, $\mathrm{BP})$ of 1-mm thickness, placed at Brewster's angle in the focused cavity arm, which acts as a Kerr medium (Fig. 1a). The effective nonlinear coefficient depends on the mode size in the BP and can be tuned via its distance from the focal plane $z_{\mathrm{BP}}$. The cavity was operated in vacuum to avoid air fluctuations and dust deposition on the mirrors. To achieve an overall anomalous roundtrip dispersion (Supplementary Fig. 6) as required for bright soliton generation, a dispersive mirror was used (see Methods). A mode-locked laser (MLL) provides a driving pulse train that is close to synchronous with the cavity. When performing small sweeps of the MLL repetition frequency $f_{\text {rep }}$ over the central cavity resonance at sufficient driving power, the bistable step in the cavity transmission signal characteristic for soliton generation ${ }^{6}$ was observed experimentally. A numerical model of the intracavity pulse propagation, based on iterative computations of an Ikeda-map-like equation system for the experimental cavity parameters (Supplementary Section I), qualitatively reproduced this feature (Fig. 1b). The high peak-power enhancement factors in the cavity predicted by the simulation (Fig. 1c) are facilitated by the spectral confinement of the loss experienced by the broad CS spectrum at the tailored ICM (Fig. 1d).

To stably maintain specific nonlinear cavity states ${ }^{23,24}$, the detuning of the MLL frequency comb from the cavity resonances at a wavelength of $1,064 \mathrm{~nm}\left(\Delta f_{1,064}\right)$ was actively stabilized with the aid of a low-power CW laser, locked to a resonance of the linear EC (Fig. 2 and Methods). The carrier-envelope offset frequency $\left(f_{0}\right)$ of the MLL is independently stabilized to an adjustable radiofrequency reference. Scans were performed by sweeping $\Delta f_{1,064}$, effectively changing $f_{\text {rep }}$ in a range of less than $1 \mathrm{~Hz}$ for constant values of $f_{0}$. Similar to recent observations in microresonators driven by pulsed lasers ${ }^{11}$, we found that the soliton step occurs only for a limited range of $f_{0}$ (Fig. $2 \mathrm{~d}$ ), corresponding to about $4 \%$ of the free

'Max-Planck-Institut für Quantenoptik, Garching, Germany. 'Ludwig-Maximilians-Universität München, Garching, Germany. ${ }^{3}$ Universität Konstanz, Department of Physics and Center for Applied Photonics, Konstanz, Germany. ${ }^{4}$ Friedrich-Schiller-Universität Jena, Institut für Angewandte Physik, Jena, Germany. ${ }^{*}$-mail: nikolai.lilienfein@mpq.mpg.de; ioachim.pupeza@mpq.mpg.de 
a
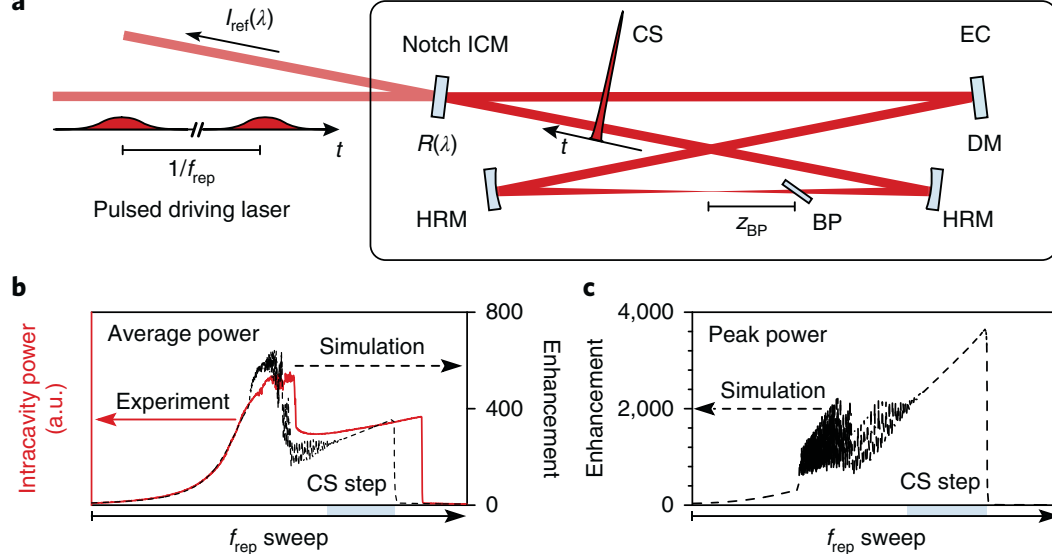

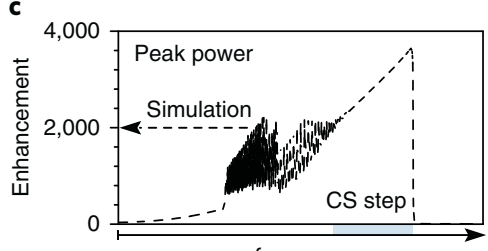

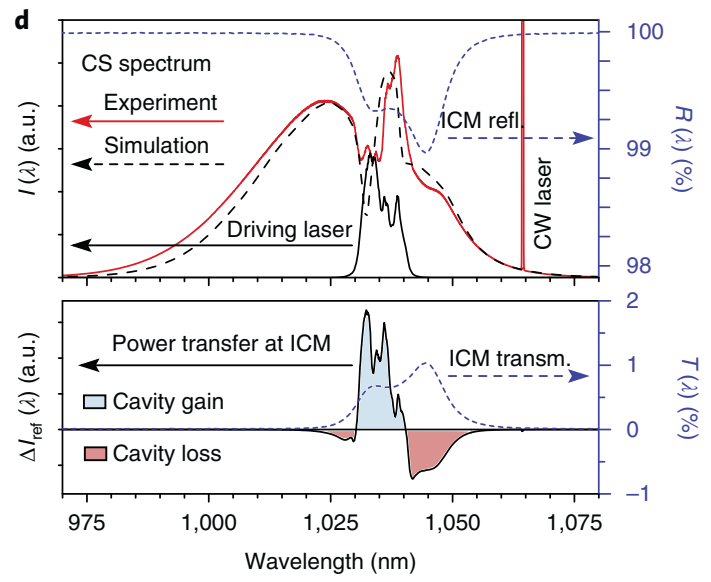

Fig. 1 | Concept for efficient CS generation. a, A train of laser pulses with a repetition rate $f_{\text {rep }}$ is coupled to a free-space EC comprising an ICM with spectrally tailored reflectivity $R(\lambda)$ (notch ICM), a dispersive mirror (DM) and two curved, highly reflective mirrors (HRM). The EC incorporates a sapphire plate arranged at Brewster's angle (BP) at a distance $z_{\mathrm{BP}}$ from the focal plane. b. Experimental intracavity power (red) and simulated enhancement factor of the average power (black), for a sweep of $f_{\text {rep }}$ from high to low frequency, exhibiting the characteristic CS step. c, Peak-power enhancement factor from the same simulation. d, Top: experimental (red) and simulated (dashed black) CS spectra, driving laser spectrum (solid black), and spectral reflectivity of the notch ICM (blue). The sharp peak at 1,064 nm is the spectrum of an auxiliary CW laser employed for the locking scheme (see Methods). d, Bottom: difference $\Delta I_{\text {ref }}(\lambda)$ between the laser spectrum and the spectrum $I_{\text {ref }}(\lambda)$ reflected from the ICM in the CS state, illustrating the high spectral overlap facilitated by the tailored transmission of the ICM. A similar scheme for spectrally tailored input coupling has previously been proposed in the context of intracavity gas plasma generation ${ }^{22}$.

a
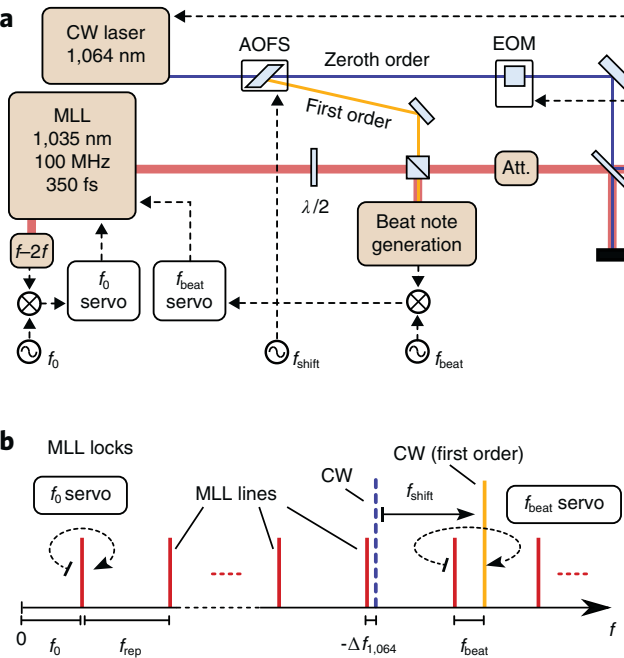

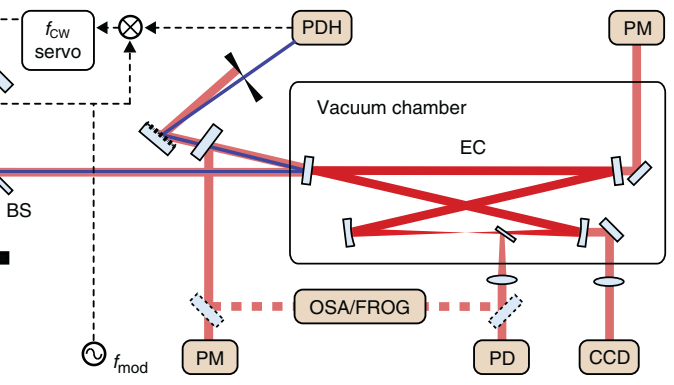

C CW-EC lock

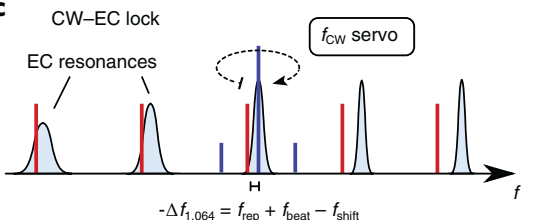

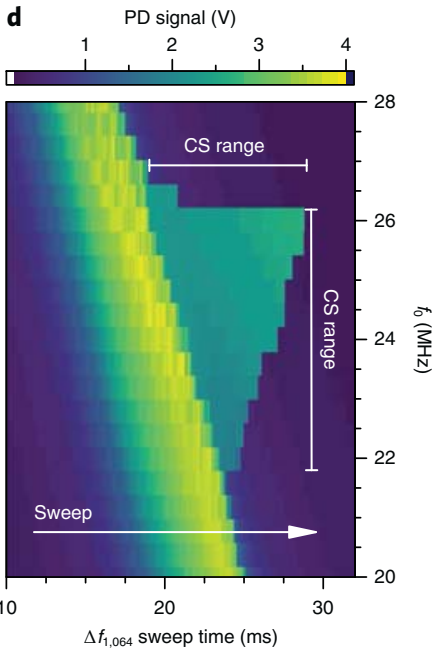

Fig. 2 | Set-up, locking scheme and $f_{0}$-dependence of the soliton regime. a, Schematic view of the locking set-up and the cavity diagnostics. The frequency $f_{\mathrm{cw}}$ of an auxilliary CW laser is locked to an EC resonance using the Pound-Drever-Hall scheme (PDH). A beatnote between the offset-frequency ( $f_{0}$ ) stabilized mode-locked driving laser (MLL) and a copy of the CW laser shifted by the frequency $f_{\text {shift }}$ is generated and locked to an adjustable reference frequency $\left(f_{\text {beat }}\right)$. The power reflected off the $E C$ is measured with a power meter (PM). For analysis of the intracavity light, the transmission through the mirrors and the reflection off the BP are used. AOFS, acousto-optic frequency shifter; Att., variable attenuator; BS, beam splitter; CCD, camera; EOM, electro-optic modulator; $f_{\text {mod }}$ modulation frequency; OSA, optical spectrum analyser; PD, photodiode. b, Frequency-domain picture of the MLL lock resulting in an adjustable detuning $\Delta f_{1,064}$ between linear cavity resonances and MLL frequency comb lines at the wavelength of 1,064 nm. c, Frequencydomain picture of the $\mathrm{CW}$-laser lock to the linear cavity resonances. The weaker lines around $f_{\mathrm{cw}}$ indicate the PDH sidebands. $\mathbf{d}$, Cavity transmission for slow blue-to-red sweeps of $\Delta f_{1,064}$ for different values of $f_{0}$, showing a strong $f_{0}$-dependence of the CS step.

spectral range of the EC. It is most pronounced at the upper edge of this range. Figure 3 shows the evolution of the intracavity spectrum during a sweep of the detuning at $f_{0}=26 \mathrm{MHz}$. At a specific detuning, the intracavity spectra change from a broad but modulated shape to smooth spectra, which increase in width with increasing detuning, a behaviour characteristic for $\mathrm{CS}^{6,24,25}$. The spectra are qualitatively reproduced by the simulation (Fig. 3 and Supplementary Fig. 7).
We characterized the temporal profile of the intracavity pulse using frequency-resolved optical gating (FROG) measurements for a CS state close to the maximum detuning, with $\Delta f_{1,030}=-0.336 \mathrm{MHz}$ being the detuning at the centre wavelength of $1,030 \mathrm{~nm}$. The retrieval yields an intracavity pulse duration of $37 \mathrm{fs}$ (Fourier limit: $36.8 \mathrm{fs}$ ), with $98 \%$ of the pulse energy carried by the main pulse (Fig. 4 and Methods). The pulse shape 


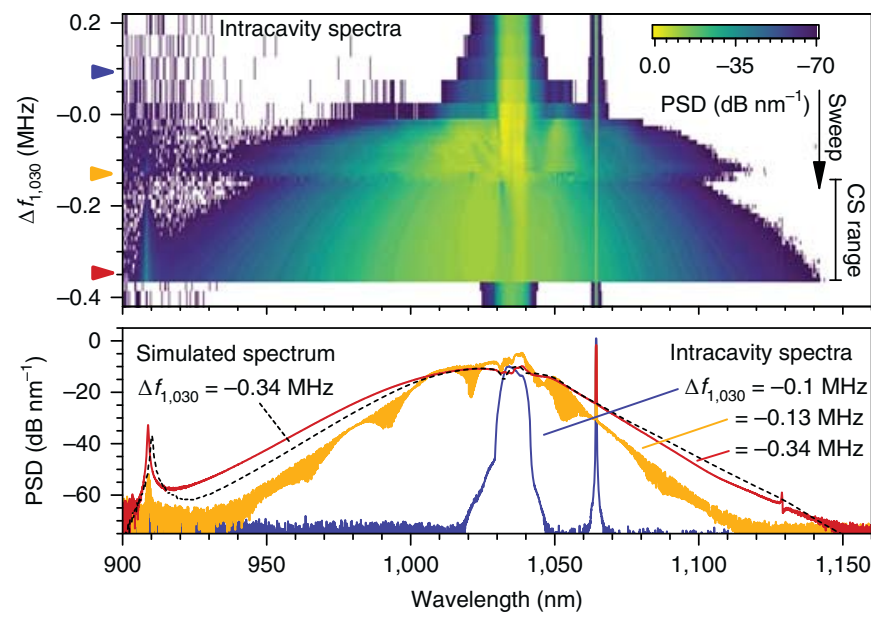

Fig. 3 | Soliton evolution. Contour plot of the experimental intracavity power spectral density (PSD) for a sweep of the detuning $\Delta f_{1,030}$, specified at a central wavelength of $1,030 \mathrm{~nm}$ (upper panel), and line cuts at three values of $\Delta f_{1,030}$ (lower panel). The cavity-enhanced CW laser is visible as a narrow peak at $1,064 \mathrm{~nm}$. The narrow peak at $910 \mathrm{~nm}$ can be associated with a dispersive wave generated via soliton Cherenkov radiation ${ }^{10}$, occurring at the zero-crossing of the cavity roundtrip phase (Supplementary Fig. 6). The simulated CS spectrum shows good agreement with the experiment, reproducing both its width and the dispersive wave.

furthermore exhibits a temporal offset between the maxima of the driving pulse and the soliton ${ }^{26,27}$. The pulse duration as well as the offset are reproduced by the model. While the FROG retrieval does not yield a time direction, the simulations reliably show temporal offsets towards the trailing edge (Supplementary Fig. 7), for a wide range of driving laser powers and values of $f_{0}$. Our simulations indicate that the sign of the temporal offset depends on the chirp, and thus the temporal asymmetry of the driving pulses, potentially in combination with the spectral asymmetry of the ICM transmission and cavity dispersion (Supplementary Section II). In this CS state, an average-power enhancement of 366 , corresponding to a peakpower enhancement of 3,220, was measured at an MLL input power of $1.6 \mathrm{~W}$ (see Methods). The linear average-power enhancement of the MLL in the same EC was 444, representing the upper limit of the linear peak-power enhancement in this EC. Notably, the soliton peak power of $125 \mathrm{MW}$ exceeds the critical power for self-focusing in sapphire by a factor of 40 . However, the short propagation length in the Kerr medium and the transverse mode guiding provided by the optically stable resonator after each pass prevent filamentation ${ }^{28}$. Calculations of the cavity caustic in the presence of a Kerr lens corresponding to the simulation results predict a beam size change in the BP of less than $10 \%$ at this power (Supplementary Section III). Similar CS states were achieved at down to two orders of magnitude lower input and intracavity power levels, limited by the focal width, by decreasing $z_{\mathrm{B} p}$. Further scaling to higher intracavity power at larger $z_{\mathrm{BP}}$ was limited by thermal drifts, probably caused by absorption in the ICM and/or the BP, preventing stable operation.

To experimentally verify the stability of the CS states, we measured the beat note between the linearly cavity-enhanced CW light and the light circulating in the MLL-driven nonlinear cavity. For a sweep of the detuning (Fig. 5a), the radio-frequency spectrum of this signal changes from a broad and noisy structure with multiple pronounced beat notes to a single, narrow peak, confirming a stable, fixed nonlinear phase shift in the soliton regime. Furthermore, we measured the relative intensity noise (RIN) of the CS state at the maximum detuning, the linear EC at low driving power and the
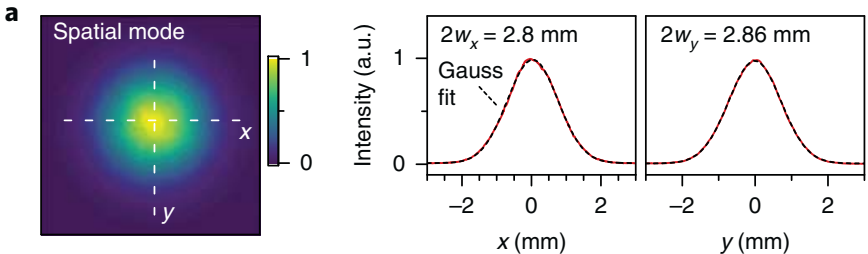

b
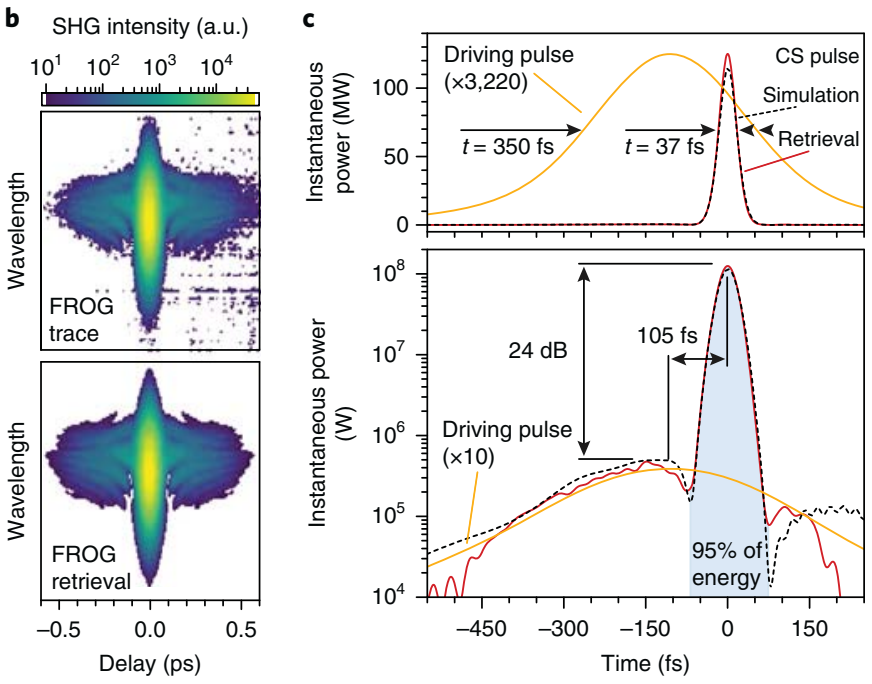

Fig. 4 | Soliton characterization. a, Spatial cavity mode profile in the CS state on the curved mirror. Line cuts (red) in the $x$ and $y$ directions are shown together with Gaussian fits (dashed black). b, Measured and retrieved second-harmonic generation (SHG) FROG traces of the CS pulse. The FROG error of the retrieval was 0.019. c, The temporal intensities of the intracavity pulse (red) and driving pulses (yellow) calculated from FROG retrieval and power measurements, and the simulated CS (dashed black) for the same driving pulse are shown on linear and logarithmic scales (upper and lower panel, respectively). The driving pulses are magnified by factors of 3,220 and 10, respectively. The temporal offset between CS and driving pulse was determined from the CS simulation (Supplementary Fig. 8).

While the agreement between simulated and experimental pulse durations is excellent, there are deviations of the peak power and of the shape of the pedestal.

driving MLL (Fig. 5b). The results show that the low-pass characteristics of the linear cavity, setting in at $500 \mathrm{kHz}$, are conserved by the CS state. More strikingly, the CS state acts as a high-pass noise filter, suppressing the RIN for frequencies below $1 \mathrm{kHz}$ by up to two orders of magnitude with respect to the driving laser and the linear cavity. This feature stems from the self-stabilizing nature of dissipative solitons in general, together with a mechanism unique to CS: the pulse energy and duration of a soliton are constrained by the balance between dispersion and nonlinear phase shift, which is fixed to the driving laser detuning. Therefore, the pulse energy and duration of the CS state is largely independent of the input power, pulse duration, or spatial overlap, as long as sufficient power to sustain the soliton is coupled to the cavity.

In conclusion, we have demonstrated the stable generation of CS in a free-space enhancement cavity with Kerr nonlinearity and a spectrally tailored input coupler transmission. We have observed intracavity pulse compression by nearly an order of magnitude, to a 37-fs, nearly Fourier-limited $\mathrm{sech}^{2}$-shaped pulse, and a low-frequency RIN-suppression mechanism of CS states. The peak-power enhancement surpasses by more than an order of magnitude the highest enhancement previously demonstrated in femtosecond enhancement cavities with comparable pulse durations ${ }^{22}$, where 

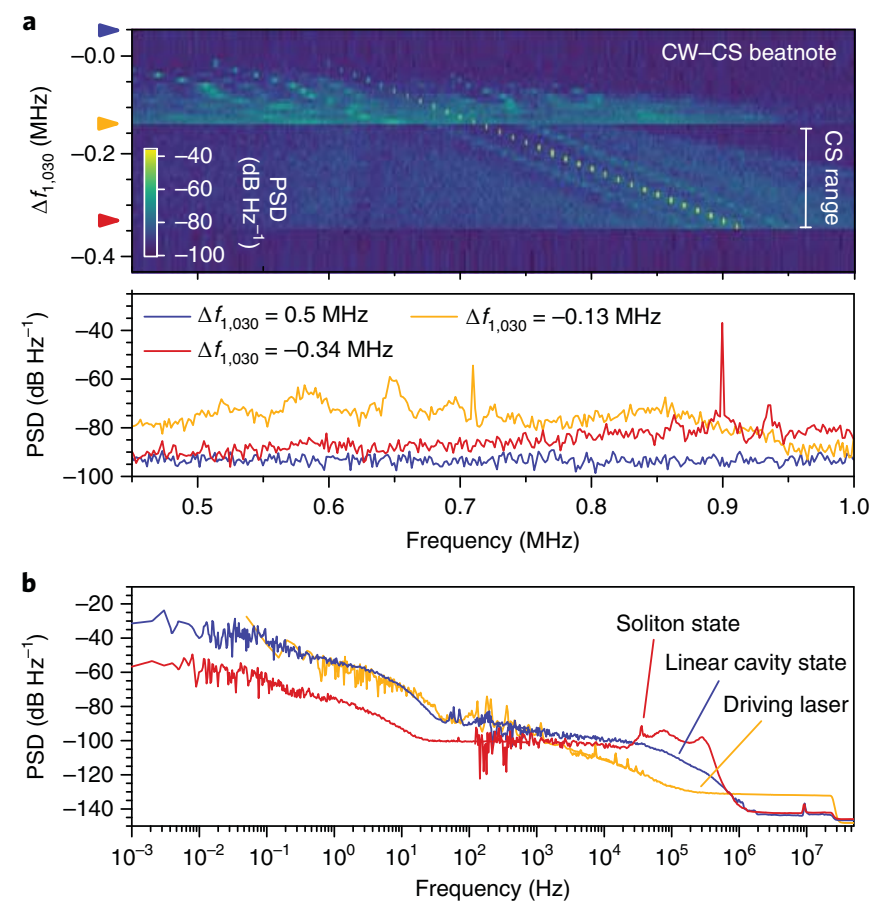

Fig. 5 | Phase and intensity stability. a, Contour plot of the radio-frequency spectrum of the beating signal between the cavity transmission of the linearly enhanced CW laser and the MLL-driven nonlinear cavity states for a sweep of the MLL detuning $\Delta f_{1,030}$ (upper panel). Line cuts at three values of $\Delta f_{1,030}$ are shown in the lower panel. $\mathbf{b}$, Power spectrum of the root mean squared (r.m.s.) RIN of the pulsed driving laser, of a (low-power) linear cavity state, and of the soliton cavity state close to maximum detuning. The frequency-resolved RIN in the CS state exhibits both EC-specific highfrequency and CS-specific low-frequency noise suppression mechanisms. Between 30 and $500 \mathrm{kHz}$, the CS RIN is significantly higher than that of both the laser and the linear cavity state. This is most likely due to CS selfstabilization dynamics excited by the phase noise of the locking loop at about $40 \mathrm{kHz}$. While the total r.m.s. RIN of $0.98 \%$ of the CS is comparable to the value of $1.05 \%$ measured for the linear cavity, the CS RIN is confined to a well-defined frequency range.

the enhancement is severely limited by the cavity-mirror dispersion $^{15,29}$. Further improvements of pulse duration, pulse energy and coupling efficiency can be expected from mirror optimization and thermal management ${ }^{30}$. Spectrally tailored input coupling could also benefit the coupling efficiency and bandwidth in microresonator geometries using multilayer mirrors ${ }^{11}$. Furthermore, we have presented a numerical model that reproduces the main experimental CS features.

Compared with previous CS platforms, free-space femtosecond ECs offer unparalleled flexibility regarding dispersion control, access to the beam, geometric scalability of the nonlinearity, and peak powers significantly exceeding the catastrophic self-focusing limit in optical materials. In this implementation, CS promise new opportunities for established applications of femtosecond ECs, such as high-repetition-rate high-order harmonic generation ${ }^{16}$ for (extreme-) ultraviolet frequency-comb ${ }^{19,20}$ and photoemission $^{31-33}$ spectroscopies, or path-length enhancement for vibrational spectroscopy ${ }^{17,18,21}$, and are likely to enable new applications. In particular, the temporal, spectral and spatial filtering, and the low-frequency RIN suppression afforded by CS are highly attractive properties for the compression of ultrashort laser pulses at the full repetition rate of their primary source.

\section{References}

1. Mollenauer, L. F. \& Stolen, R. H. The soliton laser. Opt. Lett. 9, 13-15 (1984).

2. Brabec, T., Spielmann, C. \& Krausz, F. Mode locking in solitary lasers. Opt. Lett. 16, 1961-1963 (1991).

3. Haus, H. A. Mode-locking of lasers. IEEE J. Sel. Top. Quant. Electron. 6, 1173-1185 (2000)

4. Grelu, P. \& Akhmediev, N. Dissipative solitons for mode-locked lasers. Nat. Photon. 6, 84-92 (2012).

5. Leo, F. et al. Temporal cavity solitons in one-dimensional Kerr media as bits in an all-optical buffer. Nat. Photon. 4, 471-476 (2010).

6. Herr, T. et al. Temporal solitons in optical microresonators. Nat. Photon. 8, 145-152 (2014).

7. Saha, K. et al. Modelocking and femtosecond pulse generation in chip-based frequency combs. Opt. Express 21, 1335-1343 (2013).

8. Liang, W. et al. High spectral purity Kerr frequency comb radio frequency photonic oscillator. Nat. Commun. 6, 7957 (2015).

9. Yi, X., Yang, Q.-F., Yang, K. Y., Suh, M.-G. \& Vahala, K. Soliton frequency comb at microwave rates in a high-Q silica microresonator. Optica 2, 1078-1085 (2015).

10. Brasch, V. et al. Photonic chip-based optical frequency comb using soliton Cherenkov radiation. Science 351, 357-360 (2016)

11. Obrzud, E., Lecomte, S. \& Herr, T. Temporal solitons in microresonators driven by optical pulses. Nat. Photon. 11, 600-607 (2017).

12. Pasquazi, A. et al. Micro-combs. A novel generation of optical sources. Phys. Rep. 729, 1-81 (2018)

13. Jang, J. K., Erkintalo, M., Murdoch, S. G. \& Coen, S. Ultraweak long-range interactions of solitons observed over astronomical distances. Nat. Photon. 7, 657-663 (2013)

14. Xue, X. et al. Mode-locked dark pulse Kerr combs in normal-dispersion microresonators. Nat. Photon. 9, 594-600 (2015).

15. Jones, R. J. \& Ye, J. Femtosecond pulse amplification by coherent addition in a passive optical cavity. Opt. Lett. 27, 1848-1850 (2002).

16. Gohle, C. et al. A frequency comb in the extreme ultraviolet. Nature 436, 234-237 (2005)

17. Adler, F., Thorpe, M. J., Cossel, K. C. \& Ye, J. Cavity-enhanced direct frequency comb spectroscopy: technology and applications. Annu. Rev. Anal. Chem. 3, 175-205 (2010).

18. Foltynowicz, A., Ban, T., Masłowski, P., Adler, F. \& Ye, J. Quantum-noiselimited optical frequency comb spectroscopy. Phys. Rev. Lett. 107, 233002 (2011)

19. Cingoz, A. et al. Direct frequency comb spectroscopy in the extreme ultraviolet. Nature 482, 68-71 (2012).

20. Benko, C. et al. Extreme ultraviolet radiation with coherence time greater than 1 s. Nat. Photon. 8, 530-536 (2014).

21. Reber, M. A. R., Chen, Y. \& Allison, T. K. Cavity-enhanced ultrafast spectroscopy: ultrafast meets ultrasensitive. Optica 3, 311-317 (2016).

22. Holzberger, S. et al. Femtosecond enhancement cavities in the nonlinear regime. Phys. Rev. Lett. 115, 23902 (2015)

23. Coen, S. \& Haelterman, M. Modulational instability induced by cavity boundary conditions in a normally dispersive optical fiber. Phys. Rev. Lett. 79, 4139-4142 (1997).

24. Lucas, E., Guo, H., Jost, J. D., Karpov, M. \& Kippenberg, T. J. Detuningdependent properties and dispersion-induced instabilities of temporal dissipative Kerr solitons in optical microresonators. Phys. Rev. A 95, 043822 (2017).

25. Coen, S. \& Erkintalo, M. Universal scaling laws of Kerr frequency combs. Opt. Lett. 38, 1790-1792 (2013).

26. Xu, Y. \& Coen, S. Experimental observation of the spontaneous breaking of the time-reversal symmetry in a synchronously pumped passive Kerr resonator. Opt. Lett. 39, 3492-3495 (2014).

27. Hendry, I. et al. Spontaneous symmetry breaking and trapping of temporal Kerr cavity solitons by pulsed or amplitude modulated driving fields. Phys. Rev. A 97, 053834 (2018).

28. Schulte, J., Sartorius, T., Weitenberg, J., Vernaleken, A. \& Russbueldt, P. Nonlinear pulse compression in a multi-pass cell. Opt. Lett. 41, 4511-4514 (2016)

29. Lilienfein, N. et al. Enhancement cavities for few-cycle pulses. Opt. Lett. 42, 271-274 (2017). 
30. Lilienfein, N. et al. Balancing of thermal lenses in enhancement cavities with transmissive elements. Opt. Lett. 40, 843-846 (2015).

31. Mills, A. K. et al. An XUV source using a femtosecond enhancement cavity for photoemission spectroscopy. Proc. SPIE 9512, 95121I (2015).

32. Corder, C. et al. Ultrafast extreme ultraviolet photoemission without space charge. Struct. Dyn. 5, 054301 (2018).

33. Saule, T. et al. High-flux ultrafast extreme-ultraviolet photoemission spectroscopy at $18.4 \mathrm{MHz}$ pulse repetition rate. Nat. Commun. https://doi.org/10.1038/s41467-019-08367-y (2019).

\section{Acknowledgements}

The authors gratefully acknowledge helpful suggestions from T. Herr and A. Apolonskiy. The authors thank S. Breitkopf and T. Buberl for assistance with the laser system, J. Gessner for phase measurements of cavity mirrors and M. Fischer for support concerning the offset-frequency stabilization. The authors thank the European Research Council (ERC) (617173), Deutsche Forschungsgemeinschaft (DFG) Excellence cluster 'Munich Centre of Advanced Photonics' (MAP) for funding.

\section{Author contributions}

N.L., C.H. and I.P. planned and coordinated the experiments. N.L. designed and performed the experiments, and analysed the data. C.H., T.S., M.H. and I.P. assisted with experiments and data analysis. M.H. and N.L. developed the model and performed the simulations. V.P. and M.T. designed and produced the cavity optics. E.F. contributed to the experimental concept. C.R. and A.L. conceived and implemented the spectral shift of the erbium oscillator for seeding the ytterbium amplifier system. J.L. designed and provided the amplifier system. N.L., M.H. and I.P. wrote the manuscript with input from all other authors. I.P. and F.K. supervised the project.

\section{Competing interests}

The authors declare no competing interests. 


\section{Methods}

Enhancement cavity. The spectral power reflectivity of the ICM coating was designed to exceed $99.9 \%$ in the band between 900 and $1,200 \mathrm{~nm}$, except for a notch centred at $1,040 \mathrm{~nm}$, with a minimum reflectivity of $99 \%$ and a half-depth width of $10 \mathrm{~nm}$ (Fig. 1a). The group-delay dispersion (GDD) of the mirror coatings was designed to approach $0 \mathrm{fs}^{2}$ for the ICM and for two of the highly reflective coatings, and $-45 \mathrm{fs}^{2}$ for the fourth, dispersive, highly reflective mirror, over the same spectral range. Spectral phase measurements of the individual coatings were performed using multipass spatial-spectral interferometry ${ }^{29}$. Together with the calculated dispersion of the BP, we calculated the roundtrip phase shown in Supplementary Fig. 7, corresponding to a GDD of about $-15 \mathrm{fs}^{2}$ in the central spectral region. A ring-down measurement of the EC at a wavelength of $1,064 \mathrm{~nm}$ yielded a finesse of 12,040. The corresponding roundtrip loss of $522 \mathrm{ppm}$ is dominated by the $\mathrm{BP}$ reflections and scattering at the mirror coatings. The cavity finesse at $1,035 \mathrm{~nm}$ is 842 , implying an ideal linear enhancement of 491 . A comparison to the experimental linear enhancement, which was measured at an input power of $20.3 \mathrm{~mW}$, yields the spatial overlap of 0.9. The EC was set up as a symmetric bowtie cavity with $600 \mathrm{~mm}$ radius of curvature of the concave mirrors and operated close to the inner edge of stability with a stability parameter of about -0.96 . The $1 / e^{2}$ intensity diameters of the mode on the curved mirrors was $2.8 \mathrm{~mm}$ and $2.86 \mathrm{~mm}$ in tangential and sagittal axes, respectively (Fig. $4 \mathrm{a}$ ). The distance of the BP to the focal plane $z_{\mathrm{BP}}$ was measured to be $67+/-2 \mathrm{~mm}$, which, accounting for the angle of the BP, yields an effective mode area of about $0.68 \mathrm{~mm}^{2}$ (Supplementary Section I). With the Kerr nonlinearity of sapphire being $n_{2}=0.32 \times 10^{-15} \mathrm{~cm}^{2} \mathrm{~W}^{-1}$, the corresponding effective nonlinear coefficient is $\gamma=2.77 \times 10^{-7}\left(\mathrm{~m}^{\star} \mathrm{W}\right)^{-1}$

Driving laser, locking scheme and soliton generation. The driving pulses with a duration of $350 \mathrm{fs}$ (Fourier limit: $340 \mathrm{fs}$ ) and a central wavelength of $1,035 \mathrm{~nm}$ were delivered at a repetition rate of $100 \mathrm{MHz}$ by an $\mathrm{Yb}$-fibre chirped-pulse amplifier. The Yb system was seeded by an Er:fibre MLL (C-Comb, Menlo Systems) operating at a wavelength of $1.55 \mu \mathrm{m}$. The frequency shift to $1,035 \mathrm{~nm}$ was performed using the electronic Kerr nonlinearity in a highly nonlinear germanosilicate fibre assembly. This step conserves the coherence of the Er:fibre front end ${ }^{34}$, resulting in a high level of operational stability ${ }^{35}$. The average power impinging on the EC was adjustable between $10 \mathrm{~mW}$ and $10 \mathrm{~W}$. The carrierenvelope-offset frequency $f_{0}$ of the MLL, measured with an integrated $f-2 f$ interferometer, was locked to the output of a radio-frequency waveform generator (Fig. 2a,b). For the cavity lock, a Nd:YAG non-planar ring oscillator CW laser (Mephisto, Coherent) was transmitted through an acousto-optic frequency shifter (AOFS). The zeroth-order transmission of the AOFS was phase modulated in a resonant electro-optic modulator (EOM) and spatially combined with the MLL using an 80:20 beam splitter, with $50 \mathrm{~mW}$ of the CW light impinging on the input coupler. In reflection from the EC, the CW beam at $1,064 \mathrm{~nm}$ was spatially isolated with a grating and used to generate a Pound-Drever-Hall error signal, allowing the stabilization of the CW-laser frequency to an EC resonance (Fig. 2a,c). The first-order diffraction of the CW laser in the AOFS, being frequency shifted by $f_{\text {shift }}=110 \pm 2 \mathrm{MHz}$, was combined with a part of the MLL light. A beat note was generated by broadening the MLL spectrum in a nonlinear fibre, subsequently cleaning the polarization, and filtering the wavelength to a narrow spectrum at $1,064 \mathrm{~nm}$, which was sent to a photodiode. The signal was mixed down with a frequency $f_{\text {lock }}=10.58 \mathrm{MHz}$, and can be locked by actuating on the repetition rate of the MLL. The detuning of the MLL frequency-comb lines from the cavity resonances $\Delta f_{1,064}$ at a wavelength of $1,064 \mathrm{~nm}$ is given by $\Delta f_{1,064}=f_{\text {shift }}-f_{\text {rep }}-f_{\text {beat }}$ (Fig. 2a,c). Soliton states were generated by sweeping $f_{\text {shift }}$ either continuously using the analog frequency-modulation input of the waveform generator driving the AOFS, or in steps of $<20 \mathrm{kHz}$ using its digital control (Supplementary Video 1). The frequency detuning at the central wavelength of $1,030 \mathrm{~nm} \Delta f_{1,030}$ was calculated using the measured detuning $\Delta f_{1,064}=-0.644 \mathrm{MHz}$, at which the linear cavity resonance of the MLL has its peak.

Diagnostics. The spectral transmission of the broadband cavity mirrors is not uniform over the wavelength range of interest. To gain direct access to the intracavity light we therefore used the reflections off the BP, which was slightly detuned from Brewster's angle for measurements of the intracavity spectrum, detuning curves, FROG traces and RIN data. Owing to the position of the BP in the focused cavity arm, both reflections from the close-to-parallel BP surfaces were spatially well separated after collimation. A wire-grid polarizer was used to filter out residual $s$-polarized light that was reflected off the BP after entering the cavity. The intracavity temporal intensity was calculated from the FROG retrieval (Fig. 4b), accounting for the material dispersion of the vacuum window, lens and wire-grid polarizer $(16 \mathrm{~mm}$ of fused silica). The high intensity contrast of the measurement was facilitated by the spatial homogeneity of the beam, characteristic for ECs. Because of the high sensitivity of the BP reflectivity with respect to the angle between the BP and the cavity beam axis, a calibration of the reflectivity with sufficient accuracy for precise intracavity power measurements would have been challenging. Instead, the intracavity power was calculated from a power measurement in transmission of a cavity mirror by accounting for its spectral transmission and for the intracavity spectrum ${ }^{29}$. To generate the beat signal shown in Fig. 5, the transmission through a cavity mirror was spectrally filtered to a narrow range around $1,064 \mathrm{~nm}$ using a grating. The resulting signal was sent to an amplified photodiode and measured using an electronic spectrum analyser. The RIN data were generated by acquiring oscilloscope traces of the signal of a fast photodiode with lengths of $1,000 \mathrm{~s}$ and $200 \mathrm{~ms}$, and stitching the resulting power spectra. For the RIN measurements of the linear cavity and CS, the driving laser power was $222 \mathrm{~mW}$ and $1.42 \mathrm{~W}$, respectively, and the laser was locked at $\Delta f_{1,064}=-0.642 \mathrm{MHz}$ and $\Delta f_{1,064}=-0.93 \mathrm{MHz}$, respectively.

\section{References}

34. Kumkar, S. et al. Femtosecond coherent seeding of a broadband Tm:fiber amplifier by an Er:fiber system. Opt. Lett. 37, 554-557 (2012).

35 . Wunram, M. et al. Ultrastable fiber amplifier delivering 145 -fs pulses with $6-\mu \mathrm{J}$ energy at 10-MHz repetition rate. Opt. Lett. 40, 823-826 (2015). 